\title{
The value of in vivo electrophysiological measurements for monitoring functional adaptation after massive small bowel resection in the rat
}

\author{
M C J Wolvekamp, N M C Durante, M A C Meyssen, J Bijman, H R de Jonge, R L Marquet, \\ E Heineman
}

\begin{abstract}
The process of functional adaptation after extensive small bowel resection is complex and imprecisely understood. In vivo electrophysiological measurements for monitoring the functional adaptive process after massive small bowel resection in Brown-Norway rats were evaluated. Rats underwent either a sham operation (SH) or a $\mathbf{9 0 \%}$ small bowel resection (SB). Standard rat chow was fed in unlimited quantities. At three or $\mathbf{1 0}$ weeks after operation, jejunal and ileal transepithelial potential differences (PD, $\mathbf{m V}$ ) were determined. Electrogenic ion transport in the villus was measured after glucose (sodium coupled active glucose absorption; PD-glu) and in the crypt, after theophylline infusion (theophylline stimulated chloride secretion; PD-theo). Biopsies were taken simultaneously. Each experimental group consisted of three to five animals. At three weeks the PD-theo and PD-glu in SB rats were significantly lower than in SH rats in both jejunal and ileal segments. At 10 weeks PDtheo and PD-glu were significantly diminished in the jejunal segment of the SB rats compared with the SH rats. The values of PD-theo and PD-glu in the ileal segments were, however, no longer different between the two groups. Three and 10 weeks after operation the length of the villi in the SB group was increased significantly compared with the SH controls. These results indicate that in the early phase of adaptation in vivo electrophysiological variables do not correlate with histological changes in the SB rats. This might be due to cell immaturity resulting from an increased rate of cell turnover or lack of intercellular tight junctions. This hypothesis is supported by a recovery of $P D$ responses in the ileum 10 weeks after resection.

(Gut 1993; 34: 637-642)
\end{abstract}

Extensive small bowel resection triggers complex adaptive changes. ${ }^{12}$ All resected patients need intravenous infusion to avoid the development of malnutrition while oral feeding is attempted. ${ }^{3}$ The considerably reduced absorptive surface can result in the short bowel syndrome, which is defined as incapability to thrive through oral nutrition. On the other hand, the remaining bowel can adapt sufficiently to provide essential nutritional support. Manipulating the outcome is difficult as the process of functional adaptation after extensive small bowel resection is not fully understood. Intestinal adaptation has been described as the proliferative, morphological, and functional response to various internal and external stimuli to maintain equilibrium in the gastrointestinal tract. ${ }^{4}$ After intestinal resection, hyperplastic changes caused by increased cell turnover in the proliferative zone of the crypt are generally seen in the enteric remnant. ${ }^{5}$ There is evidence that the unchanged number of cells per unit length may indicate immature enterocytes, ${ }^{5-7}$ but increased absorptive capacity per unit length has been reported as well. ${ }^{89}$ These divergent results may be ascribed to chronological differences in the studies performed. Urban and Michel postulated that morphological growth precedes functional components of intestinal adaptation after resection. ${ }^{10}$ The exact interrelation between histological changes and function, possibly regulated by different factors that may be sequentially interrelated, is unknown. The purpose of this study was to determine the value of in vivo electrophysiological measurements for monitoring functional intestinal adaptation. Electrophysiological responses are reliable indicators of small bowel function. ${ }^{11}$ We developed an in vivo technique, measuring transepithelial potential differences (PD) evoked by sodium coupled active glucose absorption, which is an index of villus function, and cyclic adenosine monophosphate (cAMP) mediated chloride secretion, predominantly reflecting crypt cell function. Moreover, histological specimens were collected to explore the interrelations between morphological and functional changes during the adaptation phases studied.

\section{Material and methods}

ANIMALS

Male rats of the inbred Brown-Norway strain (Harlan CPB, Zeist, The Netherlands) were used. The animals weighed $250-400 \mathrm{~g}$ and were bred under specific pathogen free conditions. They underwent either a sham operation (SH), a $90 \%$ small bowel resection (SB), or were control animals (IS). During the experimental period, all animals were kept under standard laboratory conditions ( 12 hours light and 12 hours dark) and were given free access to water and standard rat chow (AM-II; Hope Farms, Woerden, The Netherlands). The experimental protocols adhered to the rules laid down in The Dutch Animal Experimentation Act (1977) and the published Guidelines on the protection of experimental animals by the Council of the EC (1986). Specific protocols were approved by the committee on animal research of the Erasmus University, Rotterdam. 

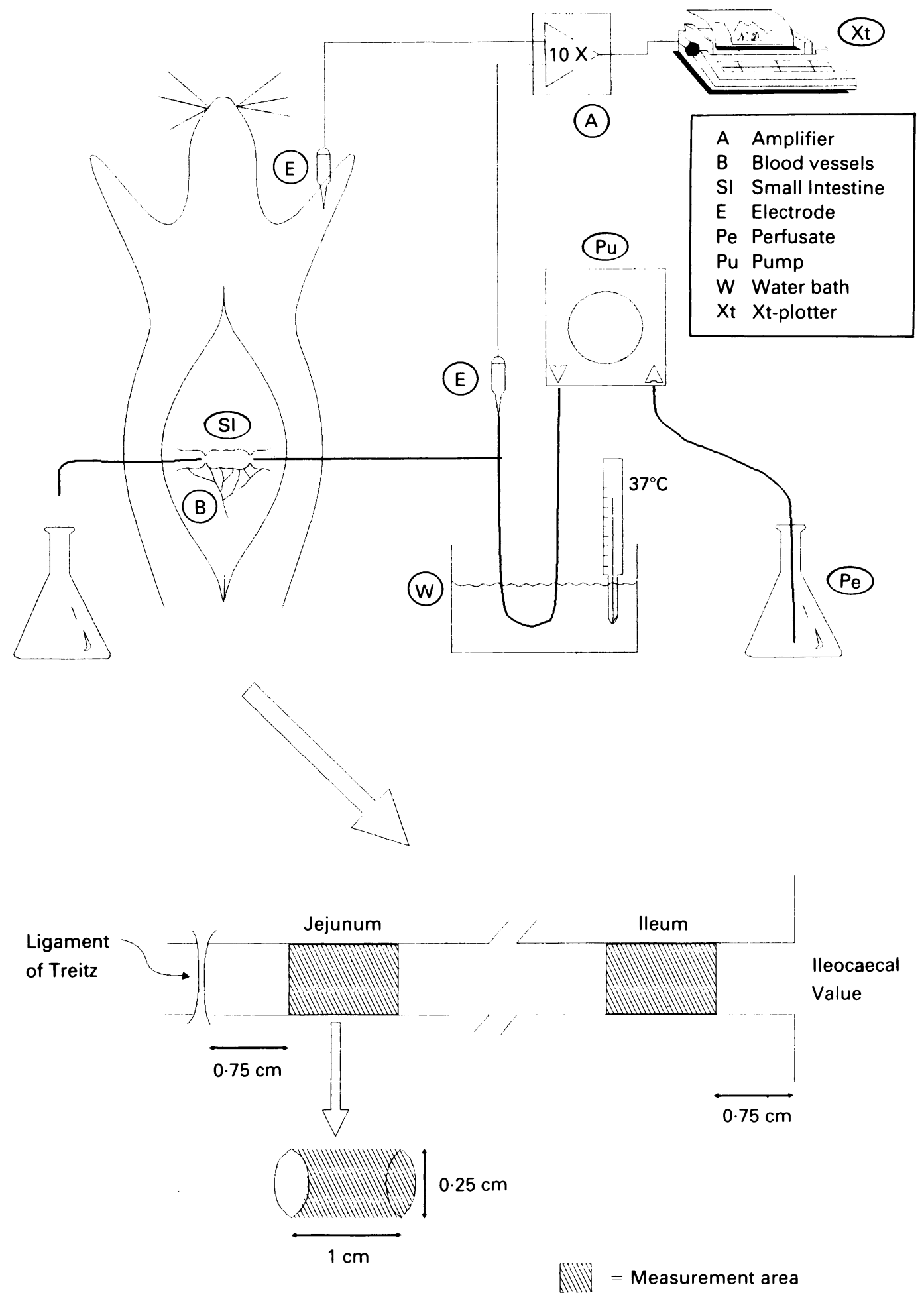

Figure 1: Overview of the set up for electrophysiological measurements.

OPERATIVE PROCEDURE

The rats were anaesthetised with ether and a midline laparotomy was performed. The SB rats were created by near total small bowel resection that is, $2.5 \mathrm{~cm}$ distally from the ligament of Trietz to $2 \cdot 5 \mathrm{~cm}$ proximally from the ileocecal valve. A sham operation was performed by transection midway between the ileum and jejunum, without removal of bowel mass. After each procedure gastrointestinal continuity was restored end to end, with Ethicon 7-0. After the operation, all animals were given $1 \mathrm{ml}$ depomycin
$20 / 20(10 \% \mathrm{v} / \mathrm{v}$ in phosphate buffered saline (PBS); Gist-Brocades, Animal Health bv, De Bilt) subcutaneously. Half of the animals in each group underwent electrophysiological monitoring and were killed three weeks after the operation $\left(\mathrm{SH}_{1}, \mathrm{n}=5\right.$ and $\left.\mathrm{SB}_{1}, \mathrm{n}=7\right)$; the other animals $\left(\mathrm{SH}_{2}, \mathrm{n}=5\right.$ and $\left.\mathrm{SB}_{2}, \mathrm{n}=5\right)$ were treated similarly 10 weeks after the operation. A third group of animals (IS, $n=3$ ) were not subjected to any operation. In these rats the superior mesenteric artery was clamped two hours before starting the electrophysiological measurement to provoke 


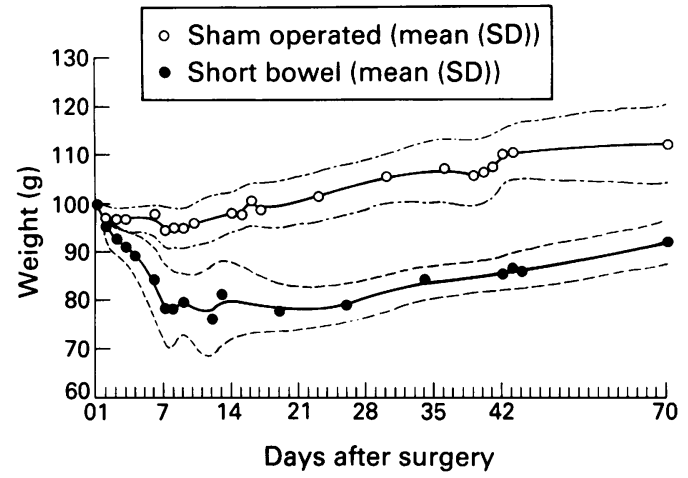

Figure 2: Weight curves.

ischaemic intestinal injury. The intestinal segment selected for measurement was anatomically identical in all rats.

\section{TECHNIQUES}

Growth assessment

The animals were weighed three times a week after the operation.

\section{Electrophysiology}

Rats were anaesthetised with ether and a midline laparotomy was performed to expose the bowel for the electrophysiological measurement. This method is a modification of the technique developed by Meyssen $e t$ al to monitor function of the small bowel in dogs. ${ }^{12}$ Well defined jejunal and ileal segment was chosen as the measurement area, in which a continuous flow of test solution was maintained with canulae. Several isoosmolar test solutions were flushed through the segment to determine intraluminal transepithelial PD with reference to a subcutaneous $\mathbf{A g}$ / $\mathrm{AgCl}_{2}$ electrode $\left(37^{\circ} \mathrm{C}, 8 \mathrm{ml} / \mathrm{min}\right)$. The standard solution consisted of: $\mathrm{NaCl}(110 \mathrm{mM})$; HEPES (5 mM); KCl (4 mM); $\mathrm{Na}_{2} \mathrm{SO}_{4}(10 \mathrm{mM})$. In the solution containing theophylline part of the mannitol was iso-osmotically replaced by $5 \mathrm{mM}$ theophylline and in the solution containing glucose, by $30 \mathrm{mM} \alpha-\mathrm{D}$-glucose. Before starting the measurement preperfusion was performed for six minutes to equilibrate luminal content with the standard perfusion solution. Then the following solutions were infused for five minutes each: standard, theophylline, standard, glucose, standard. The standard solution was used to assess the basal PDs, which reflect physiologically active ion transport. Infusion of theophylline solution $(5 \mathrm{mM})$ evoked chloride $(\mathrm{Cl}-1)$ secretion, predominantly a crypt function, resulting in an intraluminal negative PD (PD-theo). A glucose solution $(30 \mathrm{mM})$ evoked sodium coupled glucose absorption, reflecting villus function, also resulting in an intraluminal negative PD (PD-glu). Figure 1 shows an overview of the technique.

\section{Histology}

Full thickness biopsies were collected just before the electrophysiological measurements, immediately fixed in $3.6 \%$ buffered formalin, then dehydrated and embedded in paraffin. Sections of 4-5 $\mu \mathrm{m}$ were stained with haematoxylin azophloxin safran. By standardised projection of sections on a screen through a light microscope, crypt and villus length could easily be measured. Villus height was measured by subtracting crypt length - that is, the shortest distance between the bottom of intestinal villi and the lamina muscularis mucosae, from mucosal height. For each rat, 10 measurements were made and the average was used in the comparative study.

Metabolic variables

During the sixth postoperative week, rats were kept for four days in metabolic cages provided with a system to collect faeces and urine and to measure food and water consumption.

\section{Statistical analysis}

Statistical analysis of data between experimental groups was performed with the Student's $t$ test. We preferred to express our data as the difference between SH and SB at each time point, because the $\mathrm{SH}$ group tended to show a decreased PD response, apparently as a consequence of the operation. Differences with $\mathrm{p}$ values $<0.05$ were considered to be significant.

\section{Results}

WEIGHT

Figure 2 shows mean weight change curves during the period after the operation for SB and SH animals. The SH animals had a small postoperative weight loss followed by weight gain to $110 \%$ of the preoperative weight.In the SB group the follow up showed a postoperative weight loss to $70 \%-80 \%$ followed by a gradual recovery to $90 \%$ of the preoperative weight. None of these rats had returned to their weight before operation by 10 weeks after operation. Two $\mathrm{SB}_{1}$ rats died during the experimental period due to clinical short bowel syndrome.

\section{ELECTROPHYSIOLOGY}

Figures 3 and 4 show all electrophysiological data from the $\mathrm{SH}$ and $\mathrm{SB}$ rats.

\section{Fejunal electrophysiological measurements}

At three weeks, PD-glu and PD-theo in the jejunal segment of $\mathrm{SB}_{1}$ rats were significantly reduced compared with $\mathrm{SH}_{1}$ rats (PD-glu $\mathrm{p}<0.02$; PD-theo $\mathrm{p}<0.05$ ). At 10 weeks the stimulated $\mathrm{PD}$ responses in $\mathrm{SB}_{2}$ rats were significantly lower in the jejunal segment compared with $\mathrm{SH}_{2}$ rats (PD-glu $\mathrm{p}<0.01$; PD-theo $\mathrm{p}<0.05$ ). In IS rats, no response was found to either of the test solutions (results not shown).

\section{Ileal electrophysiological measurements}

At three weeks, stimulated PD responses in the ileal segment of rats from the $\mathrm{SB}_{1}$ group were significantly less for both PD-glu $(p<0.01)$ and PD-theo $(\mathrm{p}<0.05)$ compared with those in 

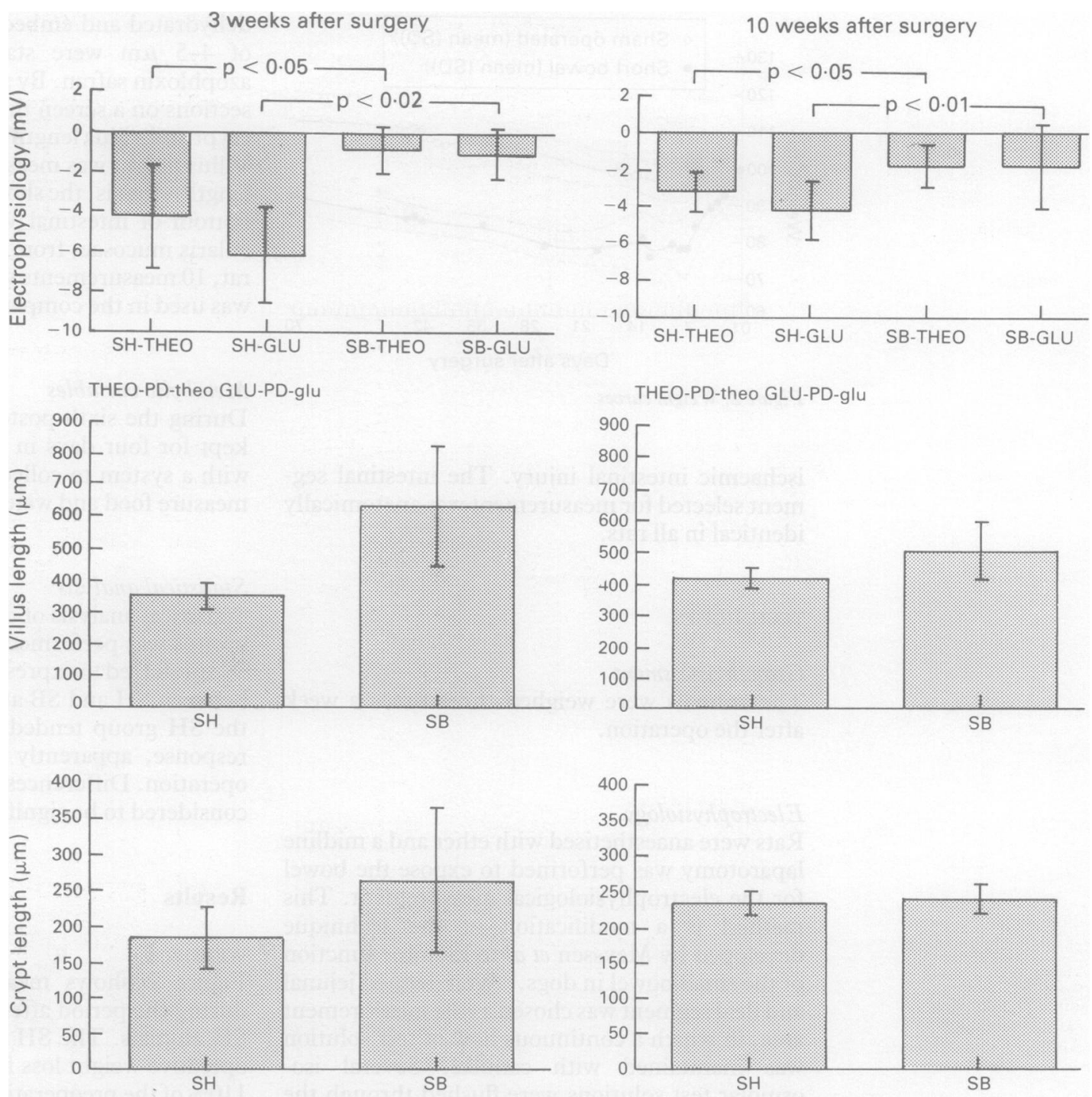

Figure 3: Fejunum: electrophysiological and histological findings. $S H$, sham operated; $S B$, short bowel; PD, potential difference; Glu, glucose; THEO, theophylline; values in mean (SD); p values calculated by Student's t test.

group $\mathrm{SH}_{1}$. At 10 weeks no significant differences in PD responses were found in either of the groups. In IS rats, again, no response was found to either of the test solutions (results not shown).

\section{HISTOLOGY}

Figures 3 and 4 show histological data.

\section{Fejunal findings}

At three weeks postoperatively, jejunal villus length was significantly enlarged in $\mathrm{SB}_{1}$ rats compared with $\mathrm{SH}_{1}$ rats. Also, jejunal villus length was significantly enlarged in $\mathrm{SB}_{2}$ rats 10 weeks after operation compared with $\mathrm{SH}_{2}$ rats. No differences in crypt length were found in either group.

\section{Ileal findings}

Three weeks after the operation, ileal villus length was significantly increased in $\mathrm{SB}_{1}$ animals compared with $\mathrm{SH}_{1}$ animals $(\mathrm{p}<0 \cdot 05)$. Ten weeks after the operation, no significant difference was found in ileal villus length between the experimental groups. Again, no differences in crypt length were found in either of the groups.

METABOLIC VARIABLES

Figure 5 shows that six weeks after the operation there were no significant differences between $\mathrm{SH}$ and SB animals for food intake, water consumption, and production of urine and faeces.

\section{Discussion}

After extensive loss of intestine both functional and morphological adaptive changes occur. Functional adaptation is determined by numerous indices, including the absorption per segment, intestinal transit time, the presence of the ileocaecal valve, intestinal contents or specific activities of brush border enzymes, and bacterial colonisation of the remaining small bowel. Increased exposure to luminal nutrients and pancreaticobiliary secretions, trophic effects of enteric hormones, and neurovascular effects on the remaining intestine are important factors that stimulate mucosal growth. Although factors regulating mucosal morphology and function of the 
3 weeks after surgery
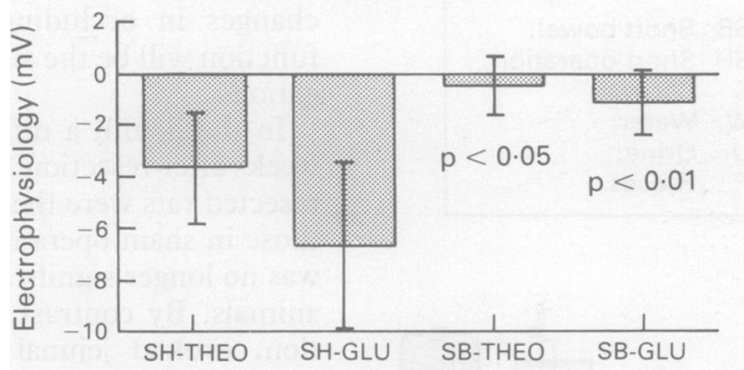

THEO-PD-theo GLU-PD-glu
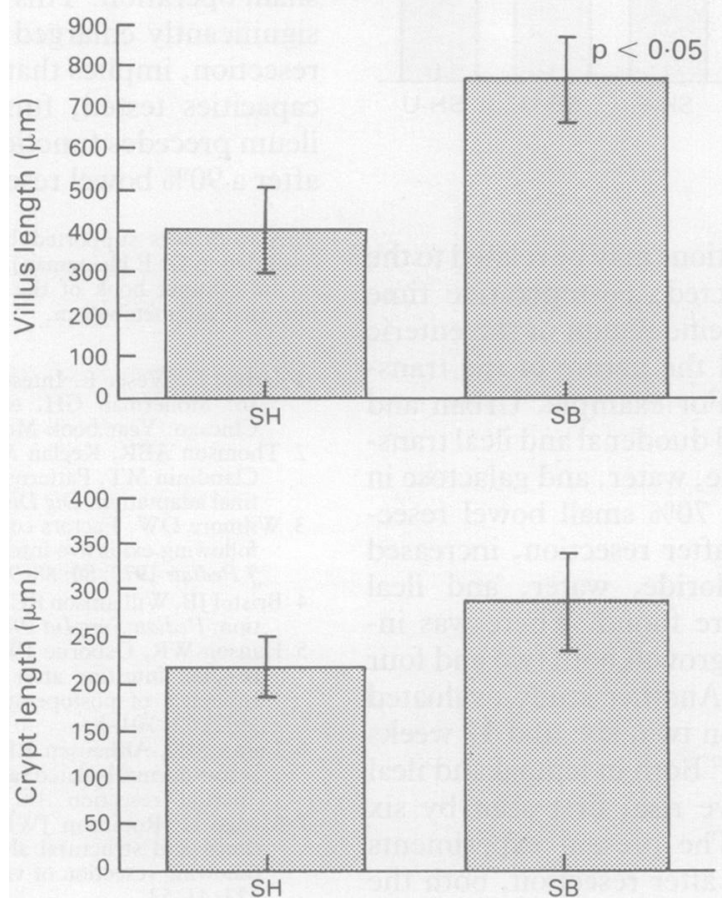

gut may be sensitively interrelated the overall process is still unravelled. The complexity of the adaptive events is indicated by region related differences in mechanisms controlling mucosal growth and function as well as the existence of dissociation between mucosal growth and components of functional adaptation. ${ }^{10}$

In our experiments we performed nearly total small bowel resection to obtain a sublethal small bowel syndrome model. In our view the occurrence of malnutrition, which is a characteristic of our sublethal model, may be a major trigger for intestinal adaptation. We set out to evaluate the interrelations between the mechanisms of active transport of electrolytes and mucosal growth in jejunal as well as ileal segments in the follow up of intestinal adaptation. The metabolic studies six weeks after operation showed that at that time differences between sham operated and resected animals were not caused by major metabolic changes. Electrophysiology, a reliable tool for functional assessment of the mechanisms of active transport of electrolytes, ${ }^{2}$ might be useful to monitor the functional adaptation process. The electrophysiological results show that the methodology developed is technically feasible in rats. It has been shown that clamping the superior mesenteric artery for two hours results in ischaemic intestinal injury in which the mechanisms of active transport of electrolytes have been damaged..$^{13}$ As expected, ischaemic control
10 weeks after surgery
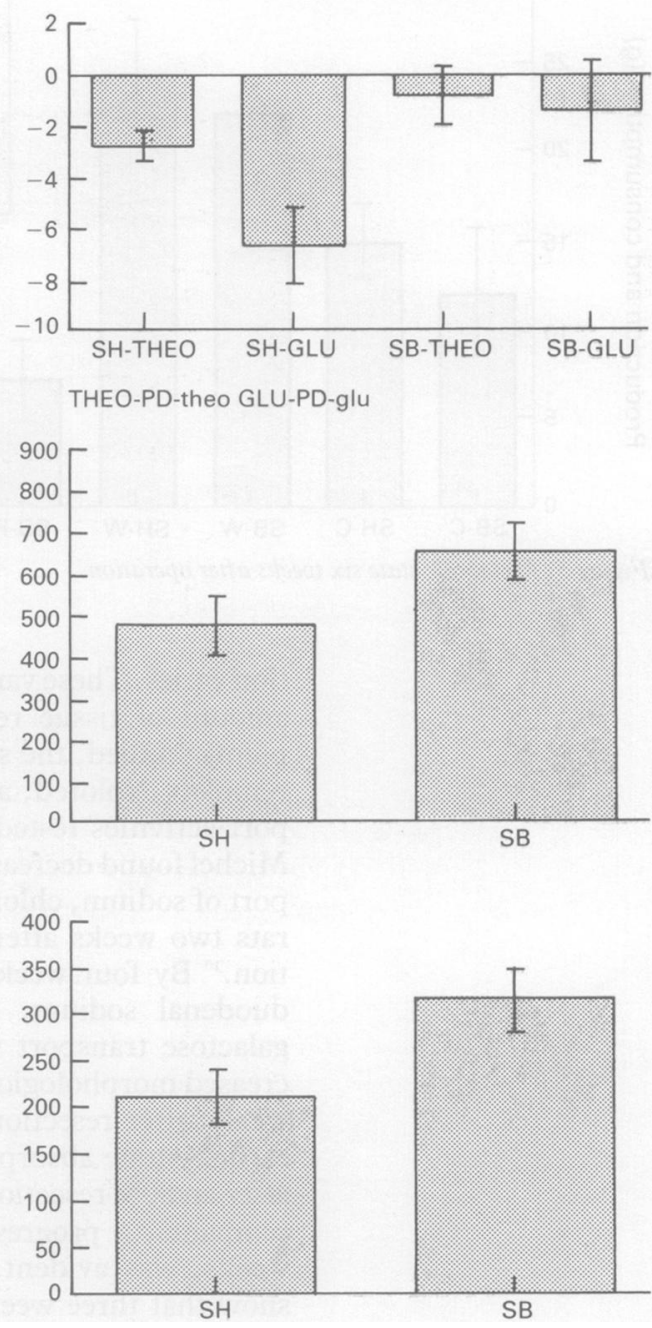

animals did not develop a transepithelial PD in response to any of the solutions. By contrast, transepithelial PD values obtained in sham operated and resected animals show that active transepithelial transport of electrolytes by an isolated bowel segment can be evaluated quantitatively by in situ electrophysiological monitoring. Previous studies have already shown that in vitro preparations of intestine can be used to study electrogenic $\mathrm{Na}^{+}$absorption and electrogenic $\mathrm{Cl}^{-}$secretion. ${ }^{14}$ From both in vitro and in vivo studies, it is generally assumed that villus epithelial cells are responsible for electrolyte coupled absorptive processes. ${ }^{14}$ is Crypt cells on the other hand, are thought to be mainly responsible for secretion of ions and water ${ }^{14}{ }^{16}$ Barry et al showed that electrical potential differences are fundamentally similar between in vivo and in vitro results. ${ }^{11}$ The in vivo technique is preferred, to avoid ischaemia, disruption of neural, lymphatic, and blood supply, and to have better sources of endogenous metabolites. The technique described in this study, however, should be refined to develop a non-invasive measuring system, as recently done for large animals. ${ }^{12}$ Such a refinement would have great value in the investigation of adaptative mechanisms by determining the effect of composition of the diet, ${ }^{18}$ hormonal supplementation, ${ }^{192}$ or manipulation of the polyamine metabolism. ${ }^{21}$ Previous experiments showed that timing of functional adapta- 


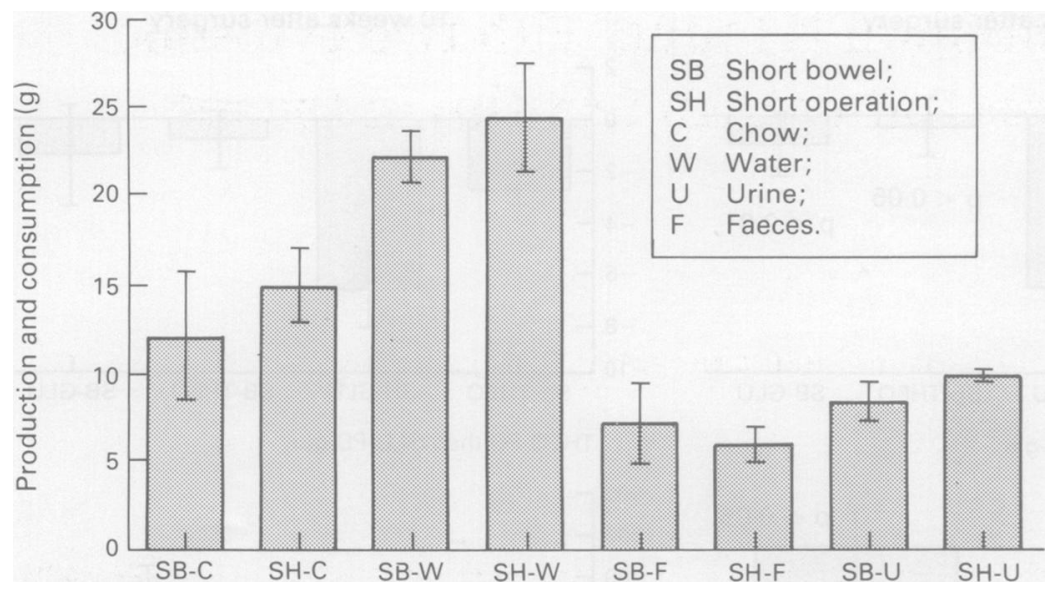

Figure 5: Metabolic state six weeks after operation.

tion varies. These variations may be related to the amount of tissue resected, postoperative time points studied, the specific region of the enteric remnant explored, and the nature of the transport activities tested. For example, Urban and Michel found decreased duodenal and ileal transport of sodium, chloride, water, and galactose in rats two weeks after a $70 \%$ small bowel resection. ${ }^{10}$ By four weeks after resection, increased duodenal sodium, chloride, water, and ileal galactose transport were found. There was increased morphological growth both two and four weeks after resection. Another study evaluated carbohydrate absorption two, six, and 12 weeks after an $80 \%$ resection..$^{22}$ Both in jejunal and ileal remnants, a progressive rise, first seen by six weeks, was evident. The present experiments show that three weeks after resection, both the glucose induced (reflecting $\mathrm{Na}^{+}$coupled glucose transport by the villus epithelium) and the theophylline provoked PD response (reflecting active $\mathrm{Cl}^{-}$secretion by the crypt epithelium) in the ileum were significantly reduced as compared with sham operation. At that time, a considerable increase in villus length was found in resected rats but no enlargement of the crypts. Similar results were found for the jejunum three weeks after resection. We assume that three weeks after resection the enteric remnant exhibits functional immaturity as a consequence of increased turnover of cells. Such immaturity has already been postulated ${ }^{5-710}$ and may explain the reduction in $\mathrm{Na}^{+}$glucose coupled transport, a characteristic feature of mature villus cells. The parallel reduction in the theophylline induced PD is more difficult to interpret, considering the prominent role of immature intestinal crypt cells in active $\mathrm{Cl}^{-}$secretion. This finding cannot be explained by a hyperactivation of the $\mathrm{Cl}^{-}$secretory pores by endogenous secretagogues in the SB group, because the basal PD was not significantly different between SH and SB rats. A possible factor expected to result in impairment of the electrical response to both glucose and theophylline in the early adaptive phase after small bowel resection might be the absence, or a functional alteration, of intercellular tight junctions during this hyperplastic period. Madara highlighted the important role of tight junctions in the function of intestinal barriers as well as the maintenance of polarity of the epithelial cells, a prerequisite for vectorial transcellular transport. ${ }^{23}$ The analysis of possible changes in occluding junction structure and function will be the subject of a separate investigation.

In the ileum, a different picture emerges 10 weeks after resection. Ileal PD measurements in resected rats were then no longer different from those in sham operated rats. Also, villus length was no longer significantly increased in resected animals. By contrast, by 10 weeks after resection, evoked jejunal PD responses remained lower compared with values 10 weeks after the sham operation. This finding, coupled with still significantly enlarged jejunal villus length after resection, implies that, for the specific transport capacities tested, functional adaptation of the ileum precedes functional adaptation of jejunum after a $90 \%$ bowel resection in rats.

This work was supported by an ESPEN research fellowship (awarded to Dr E Heineman). It was previously published in par in the abstract book of the 13th ESPEN congress on clinica nutrition and metabolism.

1 Urban E, Weser E. Intestinal adaptation to bowel resection. In: Stollerman GH, ed. Advances in Internal Medicine. Chicago: Year book Medical Publishers 1980; 26: 265-91.

2 Thomson ABR, Keelan M, Sigalet D, Fedorak R, Garg M, Clandinin MT. Patterns, mechanisms and signals for intestinal adaptation. Dig Dis 1990; 8: 99-111.

3 Wilmore DW. Factors correlating with a successful outcome following extensive intestinal resection in newborn infants. f Pediatr 1972; 80: 88-95.

4 Bristol JB, Williamson RCN. Mechanisms of intestinal adaptation. Pediatr Surg Int 1988; 4: 233-41.

5 Hanson WR, Osborne JW, Sharp JG. Compensation by the residual intestine after intestinal resection in the rat. II Influence of postoperative time interval. Gastroenterology 1977; 72: 701-5.

6 Loran MR, Althausen RL. Transport of vitamin A in vitro across normal isolated rat intestine and intestine subjected to "partial" resection. Am f Physiol 1959; 197: 1333-6.

7 Menge H, Robinson JWL. The relationship between functional and structural alterations in the rat small intestine following resection of varying extents. Res Exp Med 1978; 173: 41-53.

8 Chaves M, Smith MW, Williamson RCN. Increased activity of digestive enzymes in ileal enterocytes adapting to proximal digestive enzymes in ileal enterocytes adapt

9 Dowling RH, Booth CC. Structural and functional changes following small intestinal resection in the rat. Clin Sci 1967 32: $139-49$.

10 Urban E, Michel AM. Separation of adaptive mucosal growth and transport after small bowel resection. Gastrointestinal and Liver Physiology 1983; 7: G295-300.

11 Keast JR. Mucosal innervation and control of water and ion transport in the intestine. Rev Physiol Biochem Pharmacol 1987; 109: 1-59.

12 Meyssen MAC, Heineman E, de Bruin RWF, Veeze HJ, Bijman J, de Jonge HR, et al. Value of in vivo electrophysiological measurements to evaluate canine small bowe autotransplants. Gut 1991; 32: 1329-35.

13 Robinson JWL, Anatoli JA, Minkovitch V. The intestinal response to ischemia. Naunyn Schiedebergs Arch Pharmacol
1966; 255: 178-91.

14 Sullivan SK, Field $\dot{M}$. Ion transport across mammalian small intestine. In: Field M, Frizzell RA, eds. Handbook of intestine. In: Field M, Frize

15 Frömter E. Electrophysiological analysis of rat renal sugar and amino acid transport. I Basic Phenomena. Pflugers Arch 1982; 393: 179-89.

16 Welsh MJ, Smith PL, Fromm M, et al. Crypts are the site of intestinal fluid and electrolyte secretion. Science 1982; 218 : 1219-21.

17 Barry RJC, Dikstein S, Matthews J, Smyth DH, Wright EM Electrical potentials associated with intestinal sugar transfer. I Physiol 1964; 172: 316-38.

18 Koruda MJ, Rolandelli RH, Settle RG, Saul SH, Rombeau JL. The effect of a pectin-supplemented elemental diet on The effect of a pectin-supplemented elemental diet on intestinal adaptation to

19 Bloom SR. Gut hormones in adaptation. Gut 1987; 28 (suppl): 31-8.

20 Bass BL, Fischer BA, Richardson C, Harmon JW. Somatostatin analogue treatment inhibits postresectional adaptation of the small bowel in rats. Am $\mathcal{F}$ Surg 1991; 161 107-12.

21 Erdman SH, Park JHY, Thompson JS, Grandjean CJ, Har MH, Vanderhoof JA. Suppression of diamine oxidase activity enhances postresection ileal proliferation in the rat. Gastroenterology 1989; 96: 1533-8.

22 Bury K. Carbohydrate digestion and absorption after massive resection. Surgery 1972; 135: 177-87.

23 Madara JL. Loosening tight junctions: lessons from the intestine. F Clin Invest 1989; 83: 1089-94. 